\title{
Relationship between Multiple Intelligences and Writing Strategies
}

\author{
Narges Moheb \\ Islamic Azad University, Shiraz, Iran \\ Mohammad S. Bagheri \\ Islamic Azad University, Shiraz, Iran
}

\begin{abstract}
This study was conducted to find the relationship between multiple intelligences and writing strategies among Iranian EFL learners. The participants in this study were 120 adult males and females studying at high and advanced levels of Iran Language Institute. They filled two questionnaires during the first sessions of spring semester of 2012. The first questionnaire was a multiple intelligences inventory surveying nine types of intelligences based on Gardner's theory and the second one was a writing skills and strategies questionnaire checking the students' use of general, before, during and after writing strategies. According to the results of a corelational analysis, "logical, existential, kinesthetic, verbal and visual intelligences" correlated with "general writing strategies". Moreover, "naturalistic, logical, kinesthetic and visual intelligences" showed relationship with "before writing strategies". The researcher found that some types of intelligences among females had relationship with some writing strategies while in the male group no relationships were found. Besides, the results of advanced levels showed more significant correlations than those of high levels. Regression analysis, on the other hand, proved that none of the intelligences could predict writing strategies separately.
\end{abstract}

Index Terms - multiple intelligences, Gardner's theory, writing strategies, EFL

\section{INTRODUCTION}

This study aimed at finding the relationship between multiple intelligences (MI) of Iranian adult EFL learners and their use of writing strategies (WS). The researcher followed the MI theory proposed by Gardner (1983) and later completed by him in 1990s. According to Gardner there exist nine distinctive types of intelligences. These intelligences are:

1. Naturalist Intelligence (Nature Smart)

2. Musical Intelligence (Musical Smart)

3. Logical-Mathematical Intelligence (Number/Reasoning Smart)

4. Existential (deep question smart)

5. Interpersonal Intelligence (People Smart)

6. Bodily-Kinesthetic Intelligence (Body Smart)

7. Linguistic Intelligence (Word Smart)

8. Intra-personal Intelligence (Self Smart)

9. Spatial Intelligence (Picture Smart)

Collins (n.d.) believes that "Writing Strategies are cognitive and meta-cognitive procedures writers use to control the production of writing". Here, the researcher tries to investigate the students' use of General, Before, During And After Writing Strategies and their relationship with their nine intelligences.

Objectives of the study

The main objective of the present study was to investigate whether there is any relationship between MI and WS among Iranian adult EFL learners. To find the effect of sex and levels of students, the researcher sought to check if any of the intelligence types have any impacts on different kinds of WS used by English learners.

Research Questions

Based on the objectives, this study sought to answer the following questions:

1. Is there any relationship between MI and WS?

2. How do the results change between high and advanced groups?

3. Is the sex of participants a significant factor?

4. Which intelligence type(s) has (have) an impact on WS used by learners?

\section{LITERATURE REVIEW}

Here, some of the major previous studies with respect to multiple intelligences and their relationship with different areas in language learning are reviewed. Then, the research on the relation between multiple intelligences and writing are discussed. 


\section{A. Studies Done on Multiple Intelligences}

A lot of investigations were conducted on the effect of multiple intelligences on different aspects of language learning, especially reading comprehension.

Ghamati (2011) studied improving reading comprehension and motivation of young Iranian EFL learners through the application of MI. The results of this study revealed that using reading activities based on the multiple intelligences theory can increase reading comprehension and it increases motivation of young EFL learners to read.

Hafez (2010) worked on the relationship between undergraduate English major students' MI and their use of reading strategies. The results obtained from the co relational procedure analysis indicated that there was a meaningful relationship between the subjects' $\mathrm{MI}$ and their use of reading strategies. Furthermore, multiple regressions showed that linguistic intelligence was the best predictor of reading strategies and the linguistic intelligence was the only intelligence that had a relationship with the students' gender.

Amiriani (2010) investigated the relationship between foreign language classroom anxiety and MI. She found that there exists a significant negative relationship between anxiety and five intelligence types, namely logical-mathematical, visual-spatial, naturalistic, interpersonal and intrapersonal intelligences while the results of the regression analysis showed that none of the five intelligences or their combinations had the power to predict the variance in anxiety.

Diravidamani \&Sundarsingh (2010) studied MI and second language learning. The article examined the use of the multiple intelligence method in teaching a second language and that applying the MI method of teaching helps encourage students' involvement in the process of language acquisition.

Yi-an (2010) worked on MI and foreign language learning to find the role of MI in language learning behavior and performance. Findings showed that MI do relate to students' learning behavior and affected their English performance to some extent.

Sadri (2007-2008) studied the relationship between MI and vocabulary learning knowledge and vocabulary learning strategies among Iranian EFL learners. The findings revealed that there is a relationship between MI and vocabulary knowledge (vocabulary breadth). Moreover, stepwise multiple regression analysis showed that linguistic verbal intelligence is the best predictor of vocabulary knowledge. With respect to the relationship between MI and vocabulary strategies, the results indicated that among five categories of strategies determination, social and memory strategies had significant relationship with bodily, natural and interpersonal intelligences respectively.

McMahon, Rose and Parks (2004) worked on MI and reading achievement. They found that Students with higher scores on logical-mathematical intelligence were more likely to demonstrate at or above grade-level reading comprehension scores compared with students who scored lower on logical-mathematical intelligence.

Burman and Evans (2003) designed an action research to improve reading skills of first grade students. With the implementation of MI and increased parental involvement, students demonstrated a substantial gain in mastery of reading vocabulary words.

Cluck and Hess (2003) investigated motivating ESL learners through MI. As a result of implementing MI and cooperative learning groups, students showed an increased motivation in class work.

Gaines and Lehmann (2002) worked on a project to find the effects of using MI on reading comprehension of graduate students. They found that the student reading comprehension improved through the use of MI.

\section{B. Studies Done on Multiple Intelligences and Writing Strategies}

Little research was done on the relationship between multiple intelligences and writing strategies. The only study found in this area was done by Shah and Thomas (2002) who worked on improving the spelling of high frequency words in daily writing through the Use of MI centers. The results revealed an increase in the ability to spell high frequency words conventionally within students' daily writing. This study shed light to a new understanding of how multiple intelligences can enhance the students' learning in all areas of the curriculum.

As it is obvious the relationship between multiple intelligences and writing strategies has been almost neglected. That is why the researcher has focused on finding this relationship to fill this gap.

\section{Methodology}

This part gives information about the method of the study. First, the participants and instruments used in this study are introduced. Then, data analysis procedures are explained.

\section{A. Participants}

The participants of this study were EFL learners of Iran Language Institute (ILI) of Shiraz. The researcher used convenience sampling. 120 students were chosen among males and females of adults' branches. Half of these students were males and the other half were females. Thirty students of the male group were studying advanced 3 and the other 30 were studying high 3 . The same is true about the female group. They aged from 14 to 33 and the mean of their age was 21 .

\section{B. Instruments}


To measure the MI of the participants, the researcher used Multiple Intelligences Inventory by McKenzie (1999). This questionnaire has 90 items and consists of 9 sections, each measuring one type of intelligences with 10 items. The reliability and validity of this questionnaire was checked through the previous studies. Cronbach's alpha showed the reliability of .9 according to Amiriani (2010). The construct validity was calculated through using factor analysis which showed there were nine factors or constructs which proved the nine segments of intelligences.

The questionnaire used to check the writing strategies used by learners was 'ESLP 82 Questionnaire: SelfAssessment of English Writing Skills and Use of Writing Strategies' which was taken from the following Internet site of Marquette University on October, 20th (2011): www.marquette.edu/oie/documentary/ESLPQuestionnaireFa08.pdf

Seventy seven items of this questionnaire were used to find WS used by EFL learners including two parts. The first part was a Self-Assessment of English Writing Skills with 36 items. The researcher used this part to find the General writing strategies used by learners. The second part dealt with the learners' use of Before, During and After writing strategies with 12, 14, and 15 items respectively. The reliability of this questionnaire was calculated through the use of Cronbach's alpha which .93 which indicates that the instrument was reliable.

The two questionnaires were given to English learners of the ILI on the second session of spring semester of 1391 (April, 2012) and they were collected during the next sessions.

\section{Data Analysis}

In order to analyze the data, the $16^{\text {th }}$ version of Statistical Package for the Social Sciences (SPSS) software was used. To find the relationship between MI and WS, a series of correlations was run. To find the effect of sex and level the researcher split the files and used correlation. Finally, linear regression was used for finding which intelligence types have more impact on writing strategies.

\section{RESUltS AND DisCUSSION}

In this part the findings of this study are presented. After answering the research questions, the similarities and differences between the results of this study and some of the major previous studies are discussed.

\section{A. Is There Any Relationship between MI and WS?}

To find the relationship between MI and General, Before, During and After Writing Strategies, Pearson correlation was carried out. According to the results, five intelligences (Logical, Existential, Kinesthetic, Verbal and Visual) were correlated with General Writing Strategies. Moreover, four intelligences (Naturalistic, Logical, Kinesthetic and Visual) were correlated with Before Writing Strategies. None of the intelligences were correlated with During or After Writing Strategies.

TABLE 1:

CORRELATIONS BETWEEN MI AND WS

\begin{tabular}{|c|c|c|c|c|c|}
\hline & & General Writing & Before Writing & During Writing & After Writing \\
\hline Naturalistic & $\begin{array}{l}\text { Pearson Correlation } \\
\text { Sig. }\end{array}$ & $\begin{array}{l}.178 \\
.052\end{array}$ & $\begin{array}{l}.180 \\
.050\end{array}$ & $\begin{array}{l}.014 \\
.881\end{array}$ & $\begin{array}{l}.163 \\
.079\end{array}$ \\
\hline Musical & $\begin{array}{l}\text { Pearson Correlation } \\
\text { Sig. }\end{array}$ & $\begin{array}{l}.026 \\
.775 \\
\end{array}$ & $\begin{array}{l}.005 \\
.954\end{array}$ & $\begin{array}{l}.000 \\
.999\end{array}$ & $\begin{array}{l}-.023 \\
.804\end{array}$ \\
\hline Logical & $\begin{array}{l}\text { Pearson Correlation } \\
\text { Sig. }\end{array}$ & $\begin{array}{l}.261 * * \\
.004\end{array}$ & $\begin{array}{l}.212 * \\
.020\end{array}$ & $\begin{array}{l}.125 \\
.173 \\
\end{array}$ & $\begin{array}{l}.063 \\
.495 \\
\end{array}$ \\
\hline Existential & $\begin{array}{l}\text { Pearson Correlation } \\
\text { Sig. }\end{array}$ & $\begin{array}{l}.263 * * \\
.004\end{array}$ & $\begin{array}{l}.048 \\
.603\end{array}$ & $\begin{array}{l}.022 \\
.809\end{array}$ & $\begin{array}{l}.112 \\
.223\end{array}$ \\
\hline Interpersonal & $\begin{array}{l}\text { Pearson Correlation } \\
\text { Sig. }\end{array}$ & $\begin{array}{l}.150 \\
.103 \\
\end{array}$ & $\begin{array}{l}.166 \\
.070 \\
\end{array}$ & $\begin{array}{l}.029 \\
.754 \\
\end{array}$ & $\begin{array}{l}.119 \\
.195 \\
\end{array}$ \\
\hline Kinesthetic & $\begin{array}{l}\text { Pearson Correlation } \\
\text { Sig. }\end{array}$ & $\begin{array}{l}.217^{*} \\
.017\end{array}$ & $\begin{array}{l}.203 * \\
.026\end{array}$ & $\begin{array}{l}.029 \\
.753\end{array}$ & $\begin{array}{l}.066 \\
.474\end{array}$ \\
\hline Verbal & $\begin{array}{l}\text { Pearson Correlation } \\
\text { Sig. }\end{array}$ & $\begin{array}{l}.266^{*} \\
.003\end{array}$ & $\begin{array}{l}.123 \\
.181\end{array}$ & $\begin{array}{l}.024 \\
.797\end{array}$ & $\begin{array}{l}.066 \\
.471\end{array}$ \\
\hline Intrapersonal & $\begin{array}{l}\text { Pearson Correlation } \\
\text { Sig. }\end{array}$ & $\begin{array}{l}.093 \\
.314\end{array}$ & $\begin{array}{l}.086 \\
.352\end{array}$ & $\begin{array}{l}-.051 \\
.581\end{array}$ & $\begin{array}{l}-.054 \\
.561\end{array}$ \\
\hline Visual & $\begin{array}{l}\text { Pearson Correlation } \\
\text { Sig. }\end{array}$ & $\begin{array}{l}.198^{*} \\
.030\end{array}$ & $\begin{array}{l}.222^{*} \\
.015\end{array}$ & $\begin{array}{l}.008 \\
.929\end{array}$ & $\begin{array}{l}.072 \\
.438\end{array}$ \\
\hline
\end{tabular}

\section{B. How Do The Results Change between Intermediate and Advanced Groups?}

To find the effect of proficiency level on the results of this study the researcher split the file and ran correlation again. According to the findings, the results in the high level showed that three types of intelligences (Existential, Kinesthetic and Verbal) were correlated with General Writing Strategies. Only one type of intelligences (Intrapersonal) had relationship with After Writing Strategies. None of the intelligences were correlated with Before and During Writing Strategies. Advanced results revealed that more intelligences were correlated with WS. Three of the intelligences (Logical, Verbal and Visual) had relationship with General Writing Strategies. Seven intelligences (Naturalistic, Musical, Logical, Interpersonal, Kinesthetic, Verbal and Visual) were correlated with Before Writing Strategies. Two of 
the intelligences (Musical and Logical) had relationship with During Writing Strategies. None of the intelligences were correlated with After Writing Strategies in the advanced group.

TABLE 2:

CORRELATIONS BETWEEN MI AND WS IN HIGH LEVEL

\begin{tabular}{|c|c|c|c|c|c|}
\hline & & General Writing & Before Writing & During Writing & After Writing \\
\hline Naturalistic & $\begin{array}{l}\text { Pearson Correlation } \\
\text { Sig. }\end{array}$ & $\begin{array}{l}.239 \\
.066\end{array}$ & $\begin{array}{l}.121 \\
.359\end{array}$ & $\begin{array}{l}.000 \\
.998\end{array}$ & $\begin{array}{l}.147 \\
.262\end{array}$ \\
\hline Musical & $\begin{array}{l}\text { Pearson Correlation } \\
\text { Sig. }\end{array}$ & $\begin{array}{l}-.050 \\
.702 \\
\end{array}$ & $\begin{array}{l}-.238 \\
.067\end{array}$ & $\begin{array}{l}-.200 \\
.126\end{array}$ & $\begin{array}{l}-.163 \\
.215\end{array}$ \\
\hline Logical & $\begin{array}{l}\text { Pearson Correlation } \\
\text { Sig. }\end{array}$ & $\begin{array}{l}.209 \\
.109\end{array}$ & $\begin{array}{l}.015 \\
.912\end{array}$ & $\begin{array}{l}-.014 \\
.913\end{array}$ & $\begin{array}{l}-. .032 \\
.808\end{array}$ \\
\hline Existential & $\begin{array}{l}\text { Pearson Correlation } \\
\text { Sig. }\end{array}$ & $\begin{array}{l}. \mathbf{4 2 7} * * \\
.001\end{array}$ & $\begin{array}{l}.070 \\
.593\end{array}$ & $\begin{array}{l}.121 \\
.357\end{array}$ & $\begin{array}{l}.146 \\
.267\end{array}$ \\
\hline Interpersonal & $\begin{array}{l}\text { Pearson Correlation } \\
\text { Sig. }\end{array}$ & $\begin{array}{l}.170 \\
.193\end{array}$ & $\begin{array}{l}.020 \\
.882\end{array}$ & $\begin{array}{l}-.012 \\
.929\end{array}$ & $\begin{array}{l}.160 \\
.223\end{array}$ \\
\hline Kinesthetic & $\begin{array}{l}\text { Pearson Correlation } \\
\text { Sig. }\end{array}$ & $\begin{array}{l}\mathbf{0 . 3 1} \\
.018\end{array}$ & $\begin{array}{l}.157 \\
.230\end{array}$ & $\begin{array}{l}.127 \\
.333\end{array}$ & $\begin{array}{l}.025 \\
.849\end{array}$ \\
\hline Verbal & $\begin{array}{l}\text { Pearson Correlation } \\
\text { Sig. }\end{array}$ & $\begin{array}{l}\mathbf{0 . 2 5} \\
.049\end{array}$ & $\begin{array}{l}.033 \\
.802\end{array}$ & $\begin{array}{l}.039 \\
.769\end{array}$ & $\begin{array}{l}.161 \\
.218\end{array}$ \\
\hline Intrapersonal & $\begin{array}{l}\text { Pearson Correlation } \\
\text { Sig. }\end{array}$ & $\begin{array}{l}-.068 \\
.608\end{array}$ & $\begin{array}{l}-.062 \\
.638\end{array}$ & $\begin{array}{l}-.133 \\
.312\end{array}$ & $\begin{array}{l}-.026 \\
.043\end{array}$ \\
\hline Visual & $\begin{array}{l}\text { Pearson Correlation } \\
\text { Sig. }\end{array}$ & $\begin{array}{l}.108 \\
.410\end{array}$ & $\begin{array}{l}.062 \\
.639\end{array}$ & $\begin{array}{l}-.086 \\
.511\end{array}$ & $\begin{array}{l}-.083 \\
.526\end{array}$ \\
\hline
\end{tabular}

TABLE 3:

CORRELATIONS BETWEEN MI AND WS IN ADVANCED LEVEL

\begin{tabular}{|c|c|c|c|c|c|}
\hline & & General Writing & Before Writing & During Writing & After Writing \\
\hline Naturalistic & $\begin{array}{l}\text { Pearson Correlation } \\
\text { Sig. }\end{array}$ & $\begin{array}{l}.008 \\
.951\end{array}$ & $\begin{array}{l}\mathbf{0 . 2 6} \\
.047\end{array}$ & $\begin{array}{l}.034 \\
.794\end{array}$ & $\begin{array}{l}.095 \\
.469\end{array}$ \\
\hline Musical & $\begin{array}{l}\text { Pearson Correlation } \\
\text { Sig. }\end{array}$ & $\begin{array}{l}.156 \\
.233 \\
\end{array}$ & $\begin{array}{l}\mathbf{0 . 3 5} \\
.006 \\
\end{array}$ & $\begin{array}{l}\mathbf{0 . 2 6} \\
.044\end{array}$ & $\begin{array}{l}.166 \\
.204\end{array}$ \\
\hline Logical & $\begin{array}{l}\text { Pearson Correlation } \\
\text { Sig. }\end{array}$ & $\begin{array}{l}\mathbf{0 . 3 3} \\
.009 \\
\end{array}$ & $\begin{array}{l}\mathbf{0 . 4 6} \\
.000\end{array}$ & $\begin{array}{l}\mathbf{0 . 2 9} \\
.024\end{array}$ & $\begin{array}{l}.156 \\
.233\end{array}$ \\
\hline Existential & $\begin{array}{l}\text { Pearson Correlation } \\
\text { Sig. }\end{array}$ & $\begin{array}{l}.003 \\
.979 \\
\end{array}$ & $\begin{array}{l}.008 \\
.949 \\
\end{array}$ & $\begin{array}{l}-.109 \\
.405\end{array}$ & $\begin{array}{l}.048 \\
.717\end{array}$ \\
\hline Interpersonal & $\begin{array}{l}\text { Pearson Correlation } \\
\text { Sig. }\end{array}$ & $\begin{array}{l}.106 \\
.421\end{array}$ & $\begin{array}{l}\mathbf{0 . 3 7} \\
.004\end{array}$ & $\begin{array}{l}.082 \\
.535\end{array}$ & $\begin{array}{l}.049 \\
.712\end{array}$ \\
\hline Kinesthetic & $\begin{array}{l}\text { Pearson Correlation } \\
\text { Sig. }\end{array}$ & $\begin{array}{l}.103 \\
.433 \\
\end{array}$ & $\begin{array}{l}\mathbf{0 . 2 6} \\
.048 \\
\end{array}$ & $\begin{array}{l}-.073 \\
.581 \\
\end{array}$ & $\begin{array}{l}.073 \\
.580 \\
\end{array}$ \\
\hline Verbal & $\begin{array}{l}\text { Pearson Correlation } \\
\text { Sig. }\end{array}$ & $\begin{array}{l}\mathbf{0 . 2 9} \\
.025\end{array}$ & $\begin{array}{l}\mathbf{0 . 2 6} \\
.042\end{array}$ & $\begin{array}{l}.002 \\
.989\end{array}$ & $\begin{array}{l}-.065 \\
.621\end{array}$ \\
\hline Intrapersonal & $\begin{array}{l}\text { Pearson Correlation } \\
\text { Sig. }\end{array}$ & $\begin{array}{l}.221 \\
.089\end{array}$ & $\begin{array}{l}.244 \\
.060\end{array}$ & $\begin{array}{l}.034 \\
.794\end{array}$ & $\begin{array}{l}.036 \\
.787\end{array}$ \\
\hline Visual & $\begin{array}{l}\text { Pearson Correlation } \\
\text { Sig. }\end{array}$ & $\begin{array}{l}\mathbf{0 . 3 1} \\
.016\end{array}$ & $\begin{array}{l}\mathbf{0 . 4 3} \\
.001\end{array}$ & $\begin{array}{l}.125 \\
.340\end{array}$ & $\begin{array}{l}.223 \\
.087\end{array}$ \\
\hline
\end{tabular}

\section{Is The Sex of Participants a Significant Factor?}

To find the role of sex on the findings of the study, sorting the cases according to sex the researcher spilt the file and carried out correlations again to find if the results would change among males and females. The results of Pearson correlation revealed that, none of the intelligences were correlated with any of the writing strategies among males. Some significant relationships were found in the female group. Four intelligences (Logical, Verbal, Interpersonal and Visual) had relationship with General Writing Strategies, two intelligences were correlated with Before Writing Strategies and one intelligence (Logical) had relationship with During Writing Strategies. 
TABLE 4:

CORRELATIONS BETWEEN MI AND WS IN MALES

\begin{tabular}{|c|c|c|c|c|c|}
\hline & & General Writing & Before Writing & During Writing & After Writing \\
\hline Naturalistic & $\begin{array}{l}\text { Pearson Correlation } \\
\text { Sig. }\end{array}$ & $\begin{array}{l}.157 \\
.232\end{array}$ & $\begin{array}{l}.146 \\
.264\end{array}$ & $\begin{array}{l}-.077 \\
.557\end{array}$ & $\begin{array}{l}.073 \\
.580\end{array}$ \\
\hline Musical & $\begin{array}{l}\text { Pearson Correlation } \\
\text { Sig. }\end{array}$ & $\begin{array}{l}-.129 \\
.324\end{array}$ & $\begin{array}{l}-.108 \\
.410\end{array}$ & $\begin{array}{l}-.120 \\
.363\end{array}$ & $\begin{array}{l}-.094 \\
.474\end{array}$ \\
\hline Logical & $\begin{array}{l}\text { Pearson Correlation } \\
\text { Sig. }\end{array}$ & $\begin{array}{l}.203 \\
.120 \\
\end{array}$ & $\begin{array}{l}.156 \\
.233 \\
\end{array}$ & $\begin{array}{l}.028 \\
.829\end{array}$ & $\begin{array}{l}.051 \\
.698\end{array}$ \\
\hline Existential & $\begin{array}{l}\text { Pearson Correlation } \\
\text { Sig. }\end{array}$ & $\begin{array}{l}.242 \\
.063\end{array}$ & $\begin{array}{l}.074 \\
.573\end{array}$ & $\begin{array}{l}.037 \\
.780\end{array}$ & $\begin{array}{l}.169 \\
.197\end{array}$ \\
\hline Interpersonal & $\begin{array}{l}\text { Pearson Correlation } \\
\text { Sig. }\end{array}$ & $\begin{array}{l}.131 \\
.317\end{array}$ & $\begin{array}{l}.089 \\
.499\end{array}$ & $\begin{array}{l}.011 \\
.933\end{array}$ & $\begin{array}{l}.049 \\
.710\end{array}$ \\
\hline Kinesthetic & $\begin{array}{l}\text { Pearson Correlation } \\
\text { Sig. }\end{array}$ & $\begin{array}{l}.184 \\
.159 \\
\end{array}$ & $\begin{array}{l}.229 \\
.083 \\
\end{array}$ & $\begin{array}{l}.080 \\
.546 \\
\end{array}$ & $\begin{array}{l}.143 \\
.274 \\
\end{array}$ \\
\hline Verbal & $\begin{array}{l}\text { Pearson Correlation } \\
\text { Sig. }\end{array}$ & $\begin{array}{l}.056 \\
.671\end{array}$ & $\begin{array}{l}.111 \\
.398\end{array}$ & $\begin{array}{l}-.072 \\
.582\end{array}$ & $\begin{array}{l}.001 \\
.992\end{array}$ \\
\hline Intrapersonal & $\begin{array}{l}\text { Pearson Correlation } \\
\text { Sig. }\end{array}$ & $\begin{array}{l}-.103 \\
.435\end{array}$ & $\begin{array}{l}.013 \\
.923\end{array}$ & $\begin{array}{l}-.185 \\
.156\end{array}$ & $\begin{array}{l}-.240 \\
.065\end{array}$ \\
\hline Visual & $\begin{array}{l}\text { Pearson Correlation } \\
\text { Sig. }\end{array}$ & $\begin{array}{l}.103 \\
.435\end{array}$ & $\begin{array}{l}.211 \\
.106\end{array}$ & $\begin{array}{l}-.119 \\
.363\end{array}$ & $\begin{array}{l}-.003 \\
.981\end{array}$ \\
\hline
\end{tabular}

TABLE 5:

CORRELATIONS BETWEEN MI AND WS IN FEMALES

\begin{tabular}{|c|c|c|c|c|c|}
\hline & & General Writing & Before Writing & During Writing & After Writing \\
\hline Naturalistic & $\begin{array}{l}\text { Pearson Correlation } \\
\text { Sig. }\end{array}$ & $\begin{array}{l}.159 \\
.224\end{array}$ & $\begin{array}{l}.203 \\
.120\end{array}$ & $\begin{array}{l}.094 \\
.475\end{array}$ & $\begin{array}{l}.235 \\
.070\end{array}$ \\
\hline Musical & $\begin{array}{l}\text { Pearson Correlation } \\
\text { Sig. }\end{array}$ & $\begin{array}{l}.151 \\
.251 \\
\end{array}$ & $\begin{array}{l}.119 \\
.363 \\
\end{array}$ & $\begin{array}{l}.115 \\
.382 \\
\end{array}$ & $\begin{array}{l}.024 \\
.858\end{array}$ \\
\hline Logical & $\begin{array}{l}\text { Pearson Correlation } \\
\text { Sig. }\end{array}$ & $\begin{array}{l}\mathbf{0 . 3 2} \\
.014\end{array}$ & $\begin{array}{l}\mathbf{0 . 2 9} \\
.026\end{array}$ & $\begin{array}{l}\mathbf{0 . 2 6} \\
.047\end{array}$ & $\begin{array}{l}.059 \\
.655\end{array}$ \\
\hline Existential & $\begin{array}{l}\text { Pearson Correlation } \\
\text { Sig. }\end{array}$ & $\begin{array}{l}.251 \\
.053\end{array}$ & $\begin{array}{l}-.028 \\
.834\end{array}$ & $\begin{array}{l}-.046 \\
.728\end{array}$ & $\begin{array}{l}.015 \\
.908\end{array}$ \\
\hline Interpersonal & $\begin{array}{l}\text { Pearson Correlation } \\
\text { Sig. }\end{array}$ & $\begin{array}{l}.196 \\
.197\end{array}$ & $\begin{array}{l}\mathbf{0 . 2 7} \\
.034\end{array}$ & $\begin{array}{l}.050 \\
.705\end{array}$ & $\begin{array}{l}.191 \\
.144\end{array}$ \\
\hline Kinesthetic & $\begin{array}{l}\text { Pearson Correlation } \\
\text { Sig. }\end{array}$ & $\begin{array}{l}.222 \\
.088\end{array}$ & $\begin{array}{l}.165 \\
.209\end{array}$ & $\begin{array}{l}-.067 \\
.612 \\
\end{array}$ & $\begin{array}{l}-.032 \\
.805 \\
\end{array}$ \\
\hline Verbal & $\begin{array}{l}\text { Pearson Correlation } \\
\text { Sig. }\end{array}$ & $\begin{array}{l}. \mathbf{4 5 0} * * \\
.000\end{array}$ & $\begin{array}{l}.100 \\
.448 \\
\end{array}$ & $\begin{array}{l}.084 \\
.522 \\
\end{array}$ & $\begin{array}{l}.090 \\
.493\end{array}$ \\
\hline Intrapersonal & $\begin{array}{l}\text { Pearson Correlation } \\
\text { Sig. }\end{array}$ & $\begin{array}{l}\mathbf{0 . 2 7} \\
.035 \\
\end{array}$ & $\begin{array}{l}.154 \\
.240 \\
\end{array}$ & $\begin{array}{l}.082 \\
.532 \\
\end{array}$ & $\begin{array}{l}.116 \\
.377 \\
\end{array}$ \\
\hline Visual & $\begin{array}{l}\text { Pearson Correlation } \\
\text { Sig. }\end{array}$ & $\begin{array}{l}\mathbf{0 . 2 8} \\
.028\end{array}$ & $\begin{array}{l}.220 \\
.092\end{array}$ & $\begin{array}{l}.163 \\
.213\end{array}$ & $\begin{array}{l}.135 \\
.305\end{array}$ \\
\hline
\end{tabular}

\section{Which Intelligence Type (S) Has (Have) an Impact on Writing Strategies Used By Learners?}

Here the researcher seeks whether the intelligences that correlated with Writing Strategies could also predict them. Since the results of the first research question showed that some of the intelligences were correlated with General and Before writing strategies, two series of Regression were run to find the possible predictions.

\section{E. Impact of Intelligences on General Writing Strategies}

First General Writing Strategies were taken as dependant variables while Logical, Existential, Kinesthetic, Verbal and Visual were taken as independent variables or predictors. Regression analysis showed $\mathrm{R}$ Squared=.125 and according to ANOVA table, the results were significant. This proves that only $12.5 \%$ of the variance in general writing strategies can be explained by the combination of six intelligences. Yet, from the coefficients results we conclude that none of the intelligence types can separately predict general writing strategies.

TABLE 6:

REGRESSION ANALYSIS FOR GENERAL WRITING STRATEGIES

\begin{tabular}{|l|l|l|l|l|}
\hline \multicolumn{4}{|c|}{ REGRESSION ANALYSIS FOR GENERAL WRITING STRATEGIES } \\
\hline 1 & $\mathrm{R}$ & R Square & Adjusted R Square & Std. Error of the Estimate \\
\hline
\end{tabular}

TABLE 7:

ANOVA RESULTS FOR GENERAL WRITING STRATEGIES

\begin{tabular}{|l|l|l|l|l|l|}
\hline Model & Sum of Squares & df & Mean Square & F & Sig. \\
\hline $1 \quad$ Regression & 4315.587 & 6 & 719.265 & 2.699 & .017 \\
\hline
\end{tabular}


TABLE 8:

COEFFICIENTS RESULTS FOR GENERAL WRITING STRATEGIES

\begin{tabular}{|c|c|c|c|c|c|}
\hline & \multicolumn{2}{|c|}{ Unstandardized Coefficients } & \multicolumn{2}{|c|}{ Standardized Coefficients } & \multirow[b]{2}{*}{ Sig. } \\
\hline Model & $\mathrm{B}$ & Std. Error & Beta & $\mathrm{t}$ & \\
\hline Naturalistic & -.009 & .095 & -.011 & -.098 & .922 \\
\hline Logical & .130 & .101 & .139 & 1.291 & .199 \\
\hline Existential & .133 & .096 & .140 & 1.383 & .169 \\
\hline Kinesthetic & .095 & .099 & .101 & .952 & .343 \\
\hline Verbal & .130 & .098 & .145 & 1.318 & .190 \\
\hline Visual & -.024 & .093 & -.031 & -.258 & .797 \\
\hline
\end{tabular}

\section{F. Impact of Intelligences on Before Writing Strategies}

In this part Before Writing Strategies were taken as dependant variables while Naturalistic, Logical, Kinesthetic and Visual were taken as independent variables or predictors. Regression analysis showed R Square $=.033$ which is very low. The ANOVA table presented no significant effect from the independent variables on the dependent variable. We conclude that the combination of intelligences cannot predict before writing strategies. On the other hand, surveying the Coefficient table, we conclude that none of the intelligence types can separately predict before writing strategies.

TABLE 9:

REGRESSION ANALYSIS FOR BEFORE WRITING STRATEGIES

\begin{tabular}{|l|l|l|l|l|}
\hline Model & R & R Square & Adjusted R Square & Std. Error of the Estimate \\
\hline 1 & .354 & $\mathbf{. 1 2 5}$ & .079 & 16.32412 \\
\hline
\end{tabular}

TABLE 10:

ANOVA RESULTS FOR BEFORE WRITING STRATEGIES

\begin{tabular}{|l|l|l|l|l|l|}
\hline Model & Sum of Squares & df & Mean Square & F & Sig. \\
\hline 1 & Regression & 561.397 & 6 & 93.566 & 1.672 \\
.134 & \\
\hline
\end{tabular}

TABLE 11:

COEFFICIENTS RESULTS FOR BEFORE WRITING STRATEGIES

\begin{tabular}{|c|c|c|c|c|c|}
\hline & \multicolumn{2}{|c|}{ Unstandardized Coefficients } & \multicolumn{2}{|c|}{ Standardized Coefficients } & \\
\hline Model & $\mathrm{B}$ & Std. Error & Beta & $\mathrm{t}$ & Sig. \\
\hline Naturalistic & .030 & .044 & .076 & .690 & .491 \\
\hline Logical & .054 & .049 & .129 & 1.167 & .246 \\
\hline Existential & -.037 & .044 & -.088 & -.0852 & .396 \\
\hline Kinesthetic & .053 & .046 & .126 & 1.161 & .248 \\
\hline Verbal & -.007 & .045 & -017 & -.151 & .880 \\
\hline Visual & .033 & .043 & .096 & .769 & .443 \\
\hline
\end{tabular}

\section{G. Discussion}

The present study found that MI have a positive relationship with WS and most of the previous studies have come to a similar conclusion. Ghamati (2011), Hafez (2010), McMahon, Rose and Parks (2004), Burman and Evans (2003) and Gaines and Lehmann (2002) all found a positive relationship between MI and Reading. Yi-an (2010), Sadri (2008), Cluck and Hess (2003) and Shah and Thomas (2002) also found a positive correlation between MI and Foreign Language Learning, Vocabulary Knowledge and Strategies, Motivating ESL Learners and Improving Spelling respectively. The only contradiction was found in the findings of Amiriani (2010) who came to a negative relationship between MI and anxiety. This study and three previous studies tried to find the prediction of MI. The researcher found that none of the intelligences could predict WS. This is in agreement with the results of Amiriani (2010) who found that none of the intelligences could predict the variance in anxiety. But Hafez (2010) and Sadri (2008) found that linguistic intelligence could predict reading strategies and vocabulary strategies respectively. This study found that sex was a significant factor which is in agreement with the results of Hafez (2010). The researcher has made a table to summarize the comparison of the most related previous studies. 
TABLE 12:

SUMMARY OF PREVIOUS STUDIES

\begin{tabular}{|c|c|c|c|c|}
\hline Topic & Researcher & Relationship & Prediction of intelligences & Effect of sex and level \\
\hline $\begin{array}{l}\text { MI \& Reading Comprehension } \\
\text { and Motivation }\end{array}$ & Ghamati (2011) & +relationship & & \\
\hline MI \& Reading Strategies & Hafez (2010) & +relationship & $\sqrt{(\text { linguistic) }}$ & $\begin{array}{l}\text { Sex(significant) } \\
\text { level(significant) }\end{array}$ \\
\hline MI \& Anxiety & Amiriani (2010) & - relationship & No Prediction & \\
\hline $\begin{array}{l}\text { MI \& Second Language } \\
\text { Learning. }\end{array}$ & $\begin{array}{l}\text { Diravidamani \&Sundarsingh } \\
(2010)\end{array}$ & +relationship & & \\
\hline $\begin{array}{l}\text { MI \& Foreign Language } \\
\text { Learning }\end{array}$ & Yi-an (2010) & +relationship & & \\
\hline $\begin{array}{l}\text { MI \& Vocabulary Knowledge } \\
\text { and Strategies }\end{array}$ & Sadri (2008) & +relationship & $\sqrt{(\text { linguistic })}$ & \\
\hline MI \& Reading Achievement & $\begin{array}{l}\text { McMahon, Rose and Parks } \\
(2004)\end{array}$ & +relationship & & \\
\hline MI \& Reading Skills & Burman and Evans (2003) & +relationship & & \\
\hline MI \& Motivating ESL Learners & Cluck and Hess (2003) & +relationship & & \\
\hline MI \& Reading Comprehension & Gaines and Lehmann (2002) & +relationship & & \\
\hline MI \& Improving Spelling & Shah and Thomas (2002) & +relationship & & \\
\hline
\end{tabular}

\section{CONCLUSION}

The researcher came to the following conclusions:

1. MI of the students have relationship with S with General and Before Writing Strategies used by learners. None of the intelligences are correlated with During or After Writing Strategies.

2. MI in advanced students have more significant relationship with WS than among high levels.

3. There exists some relationship between some of intelligences and some WS used by females Interestingly, none of the intelligences are correlated with any of WS among males.

4. Although the combination of intelligences can slightly predict general writing strategies none of the intelligences can separately predict any of WS.

Pedagogical Implications

The findings of this study can make the researchers, managers, material designers, and teachers aware of how multiple intelligences could influence WS used by learners which proves the individuality of the students. This encourages them to take the necessity of using a variety of ways in teaching into a more careful consideration. The teachers are more likely to care about the strength and weakness of different intelligences among different students when teaching. Being exposed to a variety of teaching ways, the amount of learning will definitely increase. Knowing about how their intelligences act, the students themselves would also know how to improve themselves most efficiently through using different types of intelligences best. Moreover, by being aware of the WS used by students, both teachers and students would know which strategies are almost learned and used most and which strategies need more emphasis and practice which is going to help improve the students' writing.

\section{REFERENCES}

[1] Amiriani, G. (2010). On the relationship between foreign language classroom anxiety and multiple intelligences. Master's thesis, Islamic Azad University, Shiraz.

[2] Burman, T. N., \& Evans, D. C. (2003). Improving reading skills through multiple intelligences and increased parental involvement. Retrieved from ERIC (accessed 15/10/2011).

[3] Cluck, M., \& Hess, D. (2003). Improving student motivation through the use of the multiple intelligences. Retrieved from ERIC (accessed 15/10/2011).

[4] Collins, J. (n.d.). Six traits writing workshop WNY School support center UB buffalo public schools. Retrieved from: http://gse.buffalo.edu/org/writingstrategies/pdffiles/1_6Traits_Intro.pdf (accessed 10/ 5/ 2012).

[5] Diravidamani, S., \& Sundarsingh, J. (2010, January). Multiple intelligences and second language learning. Language in India, 10, 346-349.

[6] ESLP 82 Questionnaire: Self-Assessment of English writing skills and use of writing strategies. Retrieved from: www.marquette.edu/oie/documents/ESLP82QuestionnaireFa08.pdf (accessed 27/9/2011).

[7] Gaines, D., \& Lehmann, D. (2002). Improving student performance in reading comprehension through the use of multiple intelligences. Retrieved from ERIC (accessed 15/10/2011).

[8] Gardner, H. (1983). Frames of mind. The theory of multiple intelligences. New York. Basic Books.

[9] Gardner, H. (1999). Intelligence reframed: multiple intelligences for the 21st century. New York: Basic Books.

[10] Gardner, H. (1999). Are there additional intelligences? The case for naturalist, spiritual, and existential intelligences. In J. Kane (Ed.), Education, information and transformation, (pp. 111-131).

[11] Ghamati, S. (2011). Improving reading comprehension and motivation of young Iranian EFL learners through the application of the multiple intelligences theory. Master's thesis, Islamic Azad University, Shiraz.

[12] Hafez, M. (2010). The relationship between undergraduate English major students' multiple intelligences and the learners' use of reading strategies .Master's thesis, Islamic Azad University, Shiraz. 
[13] McKenzie. W. (2005). Multiple Intelligences and Instructional Technology. International Society for Technology in Education. Eugene, Oregon.

[14] McMahon, S. D., Rose, D.S., \& Parks, M. (2004, Fall). Multiple Intelligences and reading achievement: an examination of the TEELE inventory of multiple intelligences. Journal of Experimental Education, 73, 41-52.

[15] Siegler, R. S. (1992). The other Alfred Binet. Developmental psychology, 28, 179-190.

[16] Shah, T., \& Thomas, A. (2002). Improving the spelling of high frequency words in daily writing through the use of multiple intelligence centers. Retrieved from ERIC (accessed 15/10/ 2011).

[17] Yi-an, H. (2010). Multiple intelligences and foreign language learning. International Journal of the Humanities, 8, 77-105.

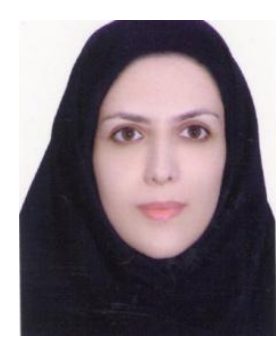

Narges Moheb holds an M.A. in TEFL. She has been Teaching English at Iran Language Institute since 2008. Ms. Moheb defended her thesis in July, 2012 under the supervision of Dr. Bagheri in Islamic Azad University of Shiraz, Iran, entitled: "Relationship between Multiple Intelligences and Writing Strategies" based on which the present paper was written. Her main research interests are multiple intelligences and learning strategies.

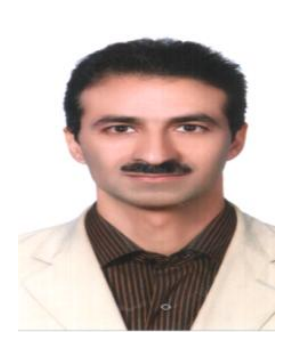

Mohammad S. Bgheri holds a PhD in TEFL. He is currently the Dean of Humanities Faculty at Islamic Azad University, Shiraz, Iran. Dr. Bagheri has published books and articles extensively and has presented a number of articles at local and international conferences. His main interests are international exams, multiple intelligences, learning strategies and assessment. 